\title{
Self-Assembled Silica Nano-Composite Polymer Electrolytes: Synthesis, Rheology \& Electrochemistry
}

\author{
Saad A. Khan \& Peter S. Fedkiw \\ Department of Chemical Engineering \\ North Carolina State University, Raleigh, NC 27695-7905 \\ Gregory L. Baker \\ Department of Chemistry \\ Michigan State University, East Lansing, MI 48824
}

\author{
A Final Report \\ Submitted to the \\ Department of Energy, Office of Basic Energy Sciences \\ DE-FG02-01ER15248
}




\section{OBJECTIVES}

The ultimate objectives of this research are to understand the principles underpinning nano-composite polymer electrolytes (CPEs) and facilitate development of novel CPEs that are low-cost, have high conductivities, large $\mathrm{Li}^{+}$transference numbers, improved electrolyte-electrode interfacial stability, yield long cycle life, exhibit mechanical stability and are easily processable.

\section{APPROACH}

Our approach is to use nanoparticulate silica fillers to formulate novel composite electrolytes consisting of surface-modified fumed silica nano-particles in polyethylene oxides (PEO) in the presence of lithium salts. We intend to design single-ion conducting silica nanoparticles which provide CPEs with high $\mathrm{Li}^{+}$transference numbers. We also will develop low-Mw (molecular weight), high-Mw and crosslinked PEO electrolytes with tunable properties in terms of conductivity, transference number, interfacial stability, processability and mechanical strength.

\section{ACCOMPLISHMENTS}

We highlight below some of our major accomplishments. Details of these can be found in our publications listed in this report.

Low-Mw PEO electrolytes.- Composite electrolytes are obtained by dispersing fumed silica particles into a liquid electrolyte (oligomeric polyethylene glycol dimethyl ether + lithium salt) forming a continuous network which mechanically supports the solution and yields a gel-like consistency. These composite electrolytes exhibit solid-like mechanical properties (elastic modulus $>10^{5} \mathrm{~Pa}$ ) while retaining the conductivity of the corresponding liquid electrolyte $\left(>10^{-3} \mathrm{~S} \mathrm{~cm}^{-1}\right.$ at $\left.25{ }^{\circ} \mathrm{C}\right)$. Lithium dendrite formation was investigated via in-situ microscopy in these low-Mw CPEs. Dendrites with a current density-dependent morphology are formed in liquid electrolytes but addition of fumed silica attenuates their formation, with hydrophilic fumed silica having a more pronounced effect than hydrophobic silica (Figure 1 and 2). We found that the dendrite inhibition effect of fumed silica is attributable to its abilities to form a continuous network with elastic-like properties and scavenge impurities from the electrolyte. We also found the presence of fumed silica in electrolytes with LiTFSi salts appeared to attenuate aluminum corrosion in cells with aluminum cathode current collector, which is very desirable in battery application.

Single-ion conducting fumed silica-based electrolytes.- In order to develop single ion conductors, we have undertaken synthetic approaches based on anchoring anions to sufficiently massive nanoparticles to ensure that anion transport is negligible. A singleion conducting fumed silica [R711-poly(lithium 2-acrylamido-2-methyl-1propanesulfonate), R711-pLiAMPS] was developed by anchoring lithium 2-acrylamido2-methyl-1-propanesulfonate (LiAMPS) onto the surface of functional fumed silica (R711) using radical copolymerization followed by ion exchange. LiAMPS is chosen as the $\mathrm{Li}^{+}$carrier because of its ease of polymerization and the highly dissociated nature of lithium sulfonates, which are electrochemically stable. Single-ion conducting CPEs were prepared by dispersing R711-pLiAMPS into low-Mw PEO. R711-pLiAMPS with a 
surface $\mathrm{Li}^{+}$content of $4.2 \mathrm{~nm}^{-2}$ provides CPEs with the highest room-temperature conductivity $\left(4.5 \times 10^{-6} \Omega \mathrm{cm}^{-1}\right.$ at $\left.\mathrm{Li}: \mathrm{O}=1: 100\right)$ and largest $\mathrm{Li}^{+}$transference number $(0.78$ at $\mathrm{Li}: \mathrm{O}=1: 20$ ). In order to increase ionic conductivity, lithium imide salt (LiTFSI) was
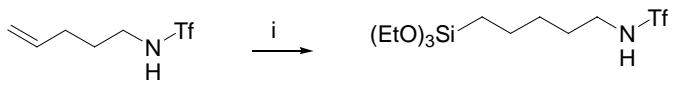

$\underline{\text { A }}$
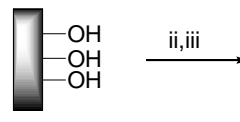

A200

Synthesis of a silica-bound TFSI analog.

i) TFA, $\mathrm{CH}_{2} \mathrm{Cl}_{2}, \mathrm{Et}_{3} \mathrm{~N}$; vi) (EtO) ${ }_{3} \mathrm{SiH}$, Karstedt's catalyst, $\mathrm{PhH}$; ii) DEA, A; iii) MeLi, toluene. added to the R711-pLiAMPS electrolyte. The addition of lithium salt greatly improves conductivity, and although the $\mathrm{Li}^{+} \quad$ transference number decreases, it remains high relative to the electrolyte with LiTFSI as the sole charge carrier (Figure 2). Single-ion conducting fumed silica-based CPEs with proper LiTFSI content in ion carriers also show higher interfacial stability than the corresponding liquid electrolyte (Figure 3).

We have also designed and synthesized fumed silica nanoparticles that have surfaces decorated with analogs of LiTFSI (Scheme 1). When dispersed in PEO, the conductivity of the CPEs range from $10^{-5}$ to $10^{-6} \mathrm{~S} / \mathrm{cm}$, surprisingly high given the two-phase nature of the PEO/silica composite and O:Li ratios >200 (Figure 4). There is a large drop in conductivity when the silica weight fraction is $>35 \%$ due to the PEO phase being noncontinuous (Figure 5). The measured $\mathrm{t}_{\mathrm{Li}}{ }^{+}$was $\sim 0.8$, in line with the conductivity being dominated by $\mathrm{Li}^{+}$transport. The conductivity shows a weak dependence on temperature, and at the same time, the TFSI analog decreased the crsytallinity of the PEO. Both observations may have important implications for low temperature electrolytes.

Crosslinked composite electrolytes.- The electrochemical and rheological properties of crosslinked CPEs based on crosslinkable fumed silica dispersed in electrolytes consisting of low-Mw PEO and LiTFSI were investigated. Various methacrylate monomers, such as methyl (MMA), ethyl (EMA), butyl (BMA), hexyl(HMA), and $n$-dodecyl (DMA) methacrylate, were added to aid in formation of the crosslinked network. We found that the presence of a crosslinked silica network does not significantly affect the mechanism of ionic transport, although the conductivity of the composites decreases by a factor of two after crosslinking with 20\% BMA (Figure 6). This suggests that the chemical crosslinks occur on a microscopic scale, as opposed to a molecular scale, between adjacent silica particles and therefore does not impede the segmental mobility of the polymer electrolyte. Increase in the length of the aliphatic chain results in both increased conductivity and mechanical strength of the crosslinkable fumed silica-based electrolyte. Conversely, increase in the weight percent of the monomer results in improved mechanical properties, but reduced conductivity.

High-Mw PEO electrolytes.- The effect of adding fumed silica on the properties of highMw PEO electrolytes was also investigated. Composite polymer electrolytes were obtained by dispersing fumed silica particles into high-Mw PEO ( Mw=200K and $600 \mathrm{~K})$ + LiTFSI. The fumed silica forms a network structure and provides mechanical support 
even when the PEO has melted. Adding fumed silica nanoparticles improves the rheological properties of polymer electrolytes, while the addition of fillers can be either beneficial or detrimental to ion-transport behavior. In crystalline polymer electrolytes (i.e., below the melting point), adding nanofillers increases the conductivity as the crystallinity decreases. In amorphous polymer electrolytes above the melting temperature, adding fillers decreases conductivity according to a volume-dilution effect. We have also found that adding fumed silica improves the stability of lithium/CPE interface and full-cell cycling performances of $\mathrm{Li} / \mathrm{CPE} / \mathrm{V}_{6} \mathrm{O}_{13}$ cells.

Mixed high- and low-Mw composite polymer electrolytes.- We undertook a novel approach to exploit the high conductivity of low-molecular weight solvents with mechanical strength of high-molecular weight polymers by using polymer blends of low and high molecular weight polyethers. The effect of temperature on rheological properties and conductivity of mixed-molecular weight composite electrolytes was investigated. Blends of PEO 600K Mw and poly(ethylene glycol)dimethyl ether (250 Mw), PEGdm(250) containing hydrophobic (R805) and hydrophilic (A200) fumed silica were prepared at a fixed ratio of high-Mw to low-Mw of $1: 1$. The concentration of fumed silica employed was $10 \mathrm{wt} \%$ and the salt (LiTFSI) was added in a 1:20 ratio. Figure 7 shows the results obtained for the blends with and without fumed silica. Hydrophilic fumed silica provides higher mechanical stability than hydrophobic fumed silica without significantly affecting the conductivity after the composite polymer electrolyte has melted. Both mixed systems containing fumed silica showed higher mechanical stability than the blend without any filler added. The results are very promising for hightemperature applications as the conductivity remains the same (and relatively high) and yet the material is mechanically stable and solid unlike traditional high molecular weight polymer systems.

Yield stress determination in nanoparticulate gels.- Appropriate yield stress determination in colloidal systems is crucial in developing composite electroyte systems and deciding on processing conditions. In this work, the magnitude of yield stress was examined for colloidal gels consisting of hydrophobic silica, polyether, and lithium salts. Serrated plates, which provide minimal wall slip, are used to compare different methods of measuring yield stress: conventional extrapolation of shear stress in steady shear experiments and dynamic experiments at large strain amplitudes. Although excellent agreement is observed in the yield stress values using both these techniques, the dynamic method seems preferable considering its experimental ease, accuracy and lack of extrapolation. Figure 8 shows an example of yield stress determination by the dynamic method; the maximum in the plot indicates the beginning of flow, and in consequence, yielding of the material.

Wall slip phenomena and surface energy effect.- Wall slip is one of the major confounding factors in estimating yield stress and processing parameters due to the fact that the no-slip boundary conditions are no longer satisfied. The occurrence of wall slip in nanoparticulate gels containing hydrophobic fumed silica (R805) and poly(ethylene glycol) dimethyl ether ( $\mathrm{Mw}=250)$, PEGdm(250) was studied by means of dynamic rheology. In this work, control of wall slip is attempted using plates modified to have 
different surface energies; in particular hydrophobic (low surface energy) and hydrophilic (high surface energy) surfaces were employed. As can be seen in Figure 9, hydrophobic plates reduce slip significantly and produce data comparable to that with the serrated plates. In contrast, hydrophilic plates have minimal effect on slip and produce data analogous to that obtained using smooth plates. These results suggest that specific interactions between the testing surface and the fumed silica particles dispersed in PEGdm(250) are dictating the slip behavior. The hydrophobic surface reduces slip possibly due to the improved association of the hydrophobic fumed silica with the wall. Figure 10 shows a schematic of the proposed mechanism for wall slip reduction with modified plates.

\section{PUBLICATIONS}

1) A. Sanchez and S.A. Khan, Effects of Temperature on Polyethers Using Mixtures of Hydrophilic and Hydrophobic Fumed Silicas as Gelling Agents, to be submitted to Langmuir.

2) Y. Li and Peter S. Fedkiw, Effect of Gel Composites Formed by Silica Nanoparticles on Aluminum Corrosion, Electrochimica Acta, 52 (2007) 2471-2477.

3) Y. Li, J. A. Yerian, H. J. Walls, and P. S. Fedkiw, Lithium Transference Number Measured by Steady State and Electrophoretic-Nuclear Magnetic Resonance (ENMR) Methods, to be submitted to Electrochemical \& Solid State Letters.

4) Yangxing Li and Peter S. Fedkiw, Rate Capabilities of Composite Gel Electrolytes Containing Fumed Silica Nanoparticles, J. Electrochem. Soc. 153 (11) A2126A2132 (2006).

5) Y. Li and P. S. Fedkiw, Nanocomposite Gel Electrolytes for Lithium-Ion Batteries, submitted to Journal of the Electrochemical Society.

6) A. Sanchez and S.A. Khan, Yield Stress vs Wall Slip in Colloidal Gels of Fumed Silica: Effects of Geometry Surface Energy on Slip Properties, Submitted to Journal of Rheology.

7) A. Sanchez and S.A. Khan, Anomalous Temperature Behavior of Fumed Silica Nanoparticulate Gels in low-MW Polyethers, Submitted to Langmuir.

8) Y. Li, J. A. Yerian, S. A. Khan, and P. S. Fedkiw, Crosslinkable Fumed SilicaBased Nanocomposite Electrolytes for Rechargeable Lithium Batteries, Journal of Power Sources 161 (2006) 1288-1296.

9) Y. Li, and P. S. Fedkiw, Rate Capabilities of Composite Gel Electrolytes Containing Fumed Silica Nanoparticles, Journal of the Electrochemical Society, 153(11) (2006) A2126-2132.

10) X.-W. Zhang, and P. S. Fedkiw, Ionic Transport and Interfacial Stability of Sulfonate-Modified Fumed Silicas as Nanocomposite Electrolytes, J. Electrochem. Soc., 152 (2005), A2413-A2420.

11) J.A. Yerian, S.A. Khan, and P. S. Fedkiw, Crosslinkable Fumed Silica-Based Nanocomposite Electrolytes: Role of Methacrylate Monomer in Formation of Crosslinked Silica Network, Journal of Power Sources, 135 (2004) 232-239.

12) X.-W. Zhang, Y. Li, S. A. Khan, and P. S. Fedkiw, Inhibition of Lithium Dendrites by Fumed Silica-Based Composite Electrolytes, Journal of Electrochemical Society, 151 (2004) A1257-A1263. 
13) X.-W Zhang, S.A. Khan, and P.S. Fedkiw, Novel Nanocomposite Electrolytes Using Single-Ion Conducting Fumed Silica, Electrochemical \& Solid-State Letters, 7 (2004) A361-A364.

14) Y. Li, X.-W. Zhang, S.A. Khan, and P.S. Fedkiw, Attenuation of Aluminum Current Collector Corrosion in LiTFSI Electrolytes using Fumed Silica Nanoparticles, Electrochemical \& Solid-State Letters, 7 (2004) A228-A230.

15) J. Zhou and Peter S. Fedkiw, "Ionic Conductivity of Composite Electrolytes Based on Oligo(Ethylene Oxide) and Fumed Oxides,” Solid State Ionics, 166(2004), 275293.

16) J. Zhou and P. S. Fedkiw, Cycling of Lithium/Metal Oxide Cells Using Composite Electrolytes Containing Fumed Silicas, Electrochimica Acta, 48 (2003) 2571-2582.

17) H.J. Walls, T.A. Zawodzinski, P.S. Fedkiw, S.A. Khan, Ion transport in Nanocomposite Electrolytes, Journal of the Electrochemical Society, 150 (2003) E165-E174.

18) H.J. Walls, M.W. Riley, P.S. Fedkiw, R.J. Spontak, S.A. Khan, Composite Electrolytes from Self-assembled Colloidal Networks", Electrochimica Acta, 48 (2003) 2071-2077.

19) H.J. Walls, M.W. Riley, R.R. Gupta, R.J. Spontak, P.S. Fedkiw, S.A. Khan, Nanocomposite Electrolytes from Fumed Silica and Hectorite Clays: Passive vs. Active Fillers, Advanced Functional Materials, 13 (2003) 710-717.

20) H.J. Walls, S.B. Caines, A.M. Sanchez, S.A. Khan, Yield Stress and Wall Slip Phenomena in Colloidal Silica Gels, Journal of Rheology, 47 (2003) 847-864.

21) Yangxing Li, Peter S. Fedkiw, and Saad A. Khan, Lithium $/ \mathrm{V}_{6} \mathrm{O}_{13}$ Cells Using Silica Nanoparticle-Based Composite Electrolyte, Electrochimica Acta, 47(2002), 3853.

22) Jian Zhou, Peter S. Fedkiw, and Saad A. Khan, Interfacial Stability Between Lithium and Fumed Silica-Based Composite Electrolytes, J. Electrochem. Soc., 149 (2002), A1121. 
(A)
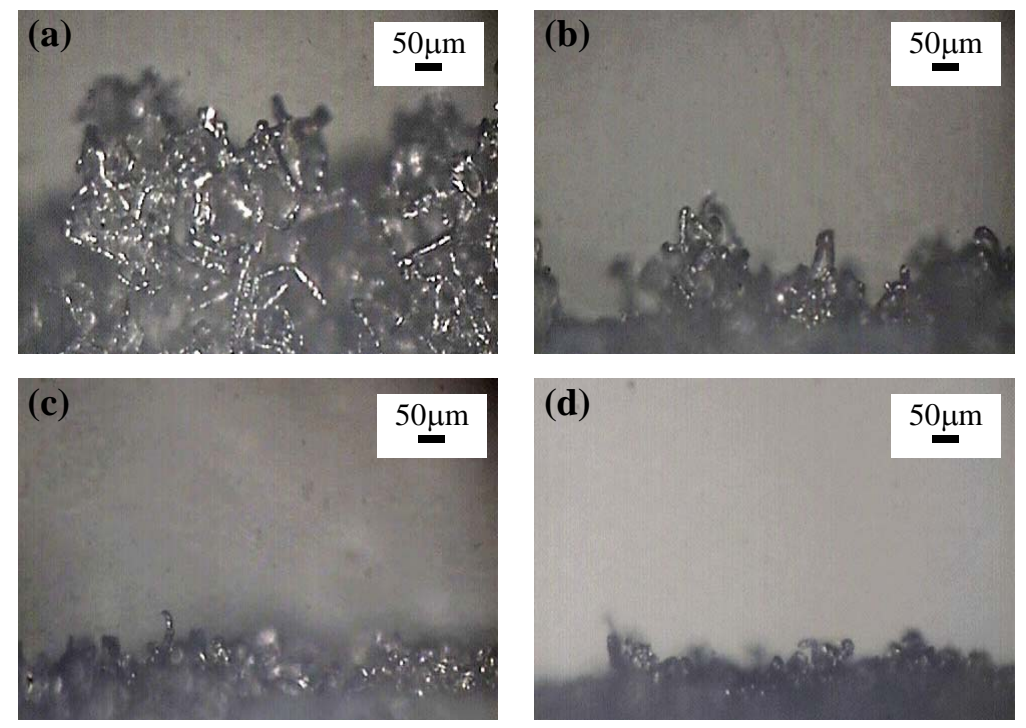

(B)
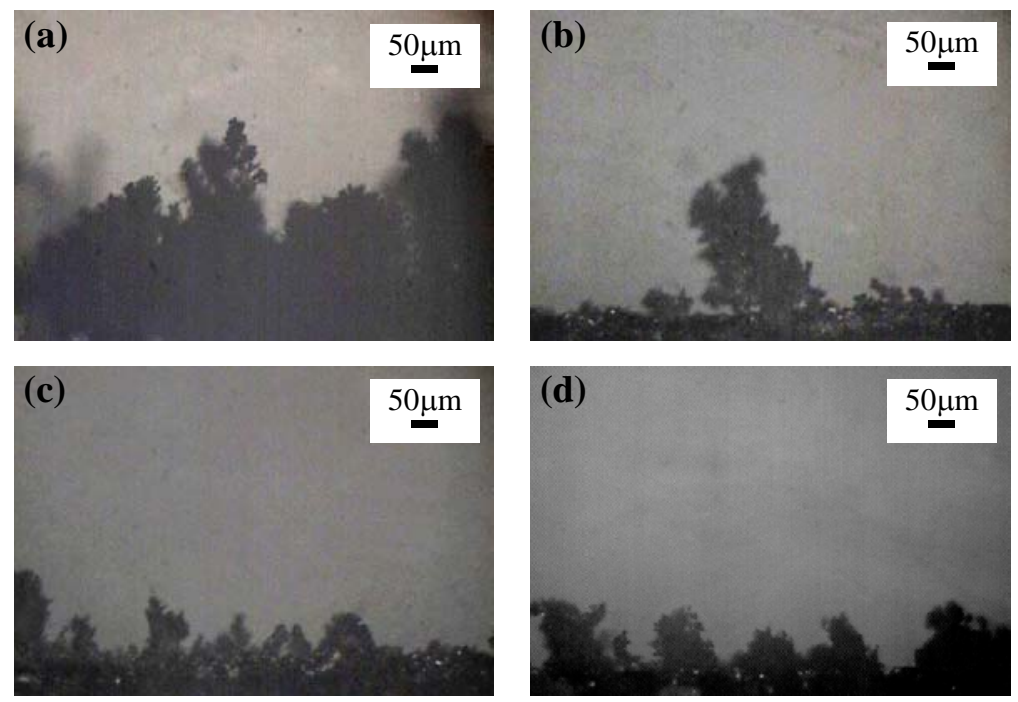

Figure 1. Micrographs of lithium dendrites $\left(3.6 \mathrm{C} \mathrm{cm}^{-2}\right)$ in fumed silica (R805)-based composite electrolytes with various filler content: (a) $0 \mathrm{wt} \%$; (b) $5 \mathrm{wt} \%$; (c) $10 \mathrm{wt} \%$; and (d) $20 \mathrm{wt} \%$. The current density was (A) $0.2 \mathrm{~mA} \mathrm{~cm}^{-2}$; (B) 1 $\mathrm{mA} \mathrm{cm}{ }^{-2}$. 


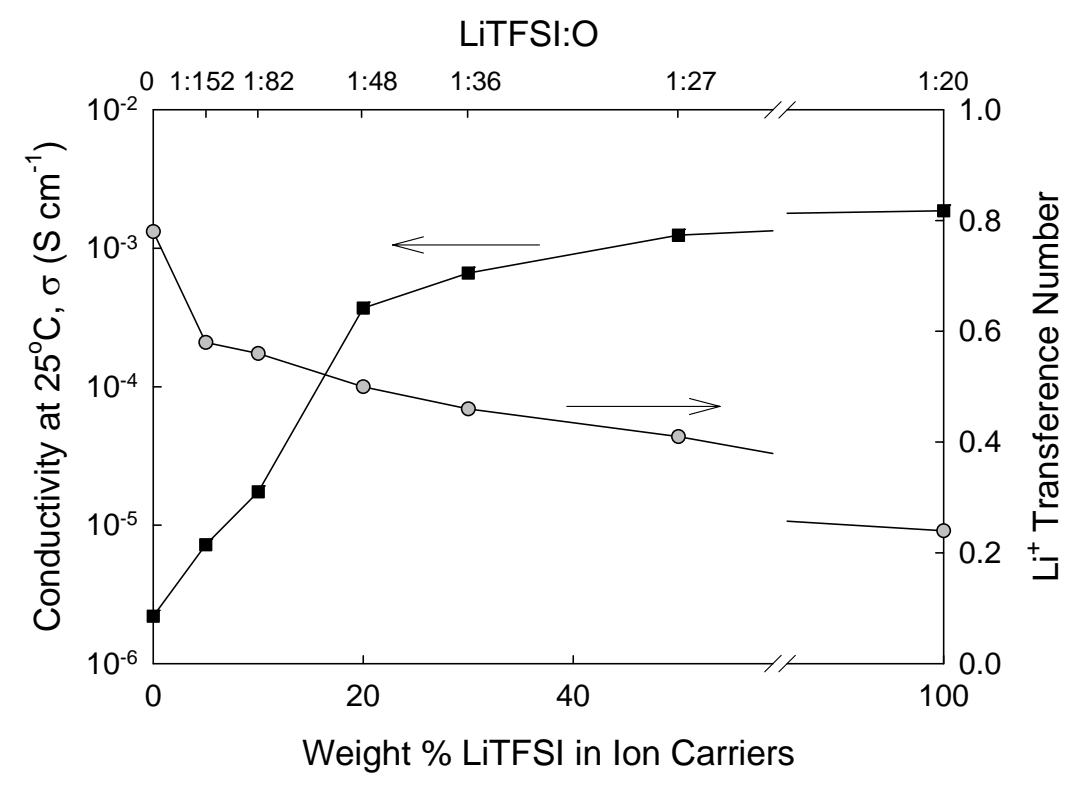

Figure 2. Room-temperature conductivity and $\mathrm{Li}^{+}$transference number of single-ion fumed silica-based CPEs as a function of LiTFSI content in ion carriers. Both R711-pLiAMPS and LiTFSI content vary such that $\mathrm{Li}$ (total):O = 1:20. At $100 \%$ LiTFSI, the electrolyte is liquid since no fumed silica is present.

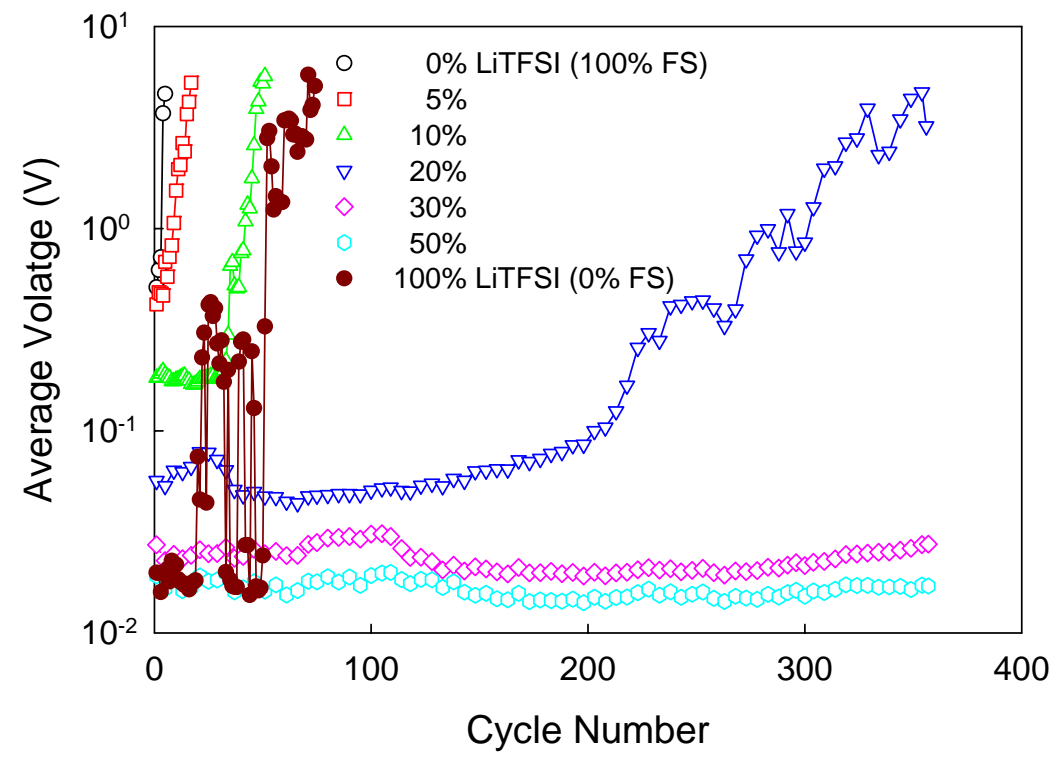

Figure 3. Interfacial stability of CPEs with various LiTFSI content. Both R711-pLiAMPS and LiTFSI content vary such that $\mathrm{Li}$ (total): $\mathrm{O}=1: 20$. Lithium cycling was conducted by applying a current of $0.05 \mathrm{~mA} \mathrm{~cm}^{-2}$ on $\mathrm{Li} / \mathrm{CPE} / \mathrm{Li}$ cells, and the current was reversed every $1 \mathrm{~h}\left(180 \mathrm{mC} \mathrm{cm}^{-2}\right)$. 


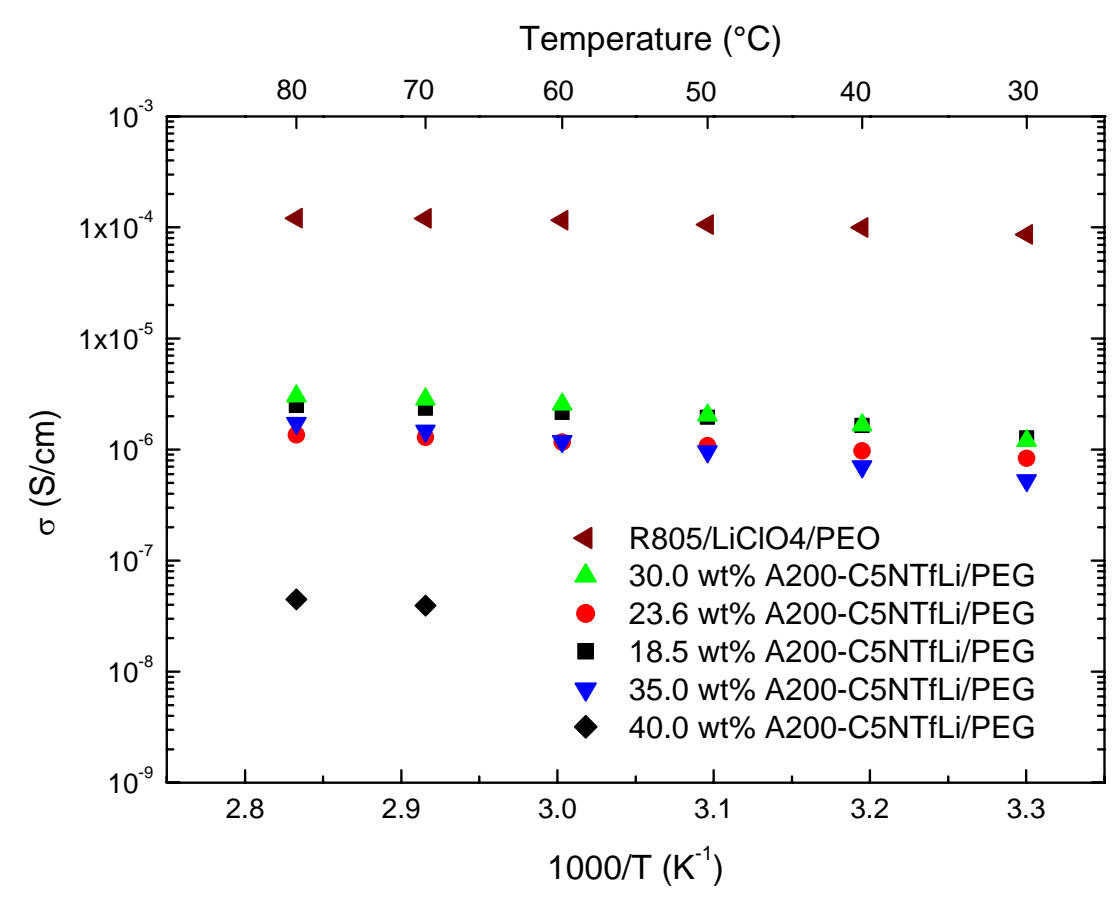

Figure 4. Temperature dependent conductivity of composites based on A200-C5NTfLi and polyethylene glycol dimethyl ether (ca. $500 \mathrm{~g} / \mathrm{mol}$ ).

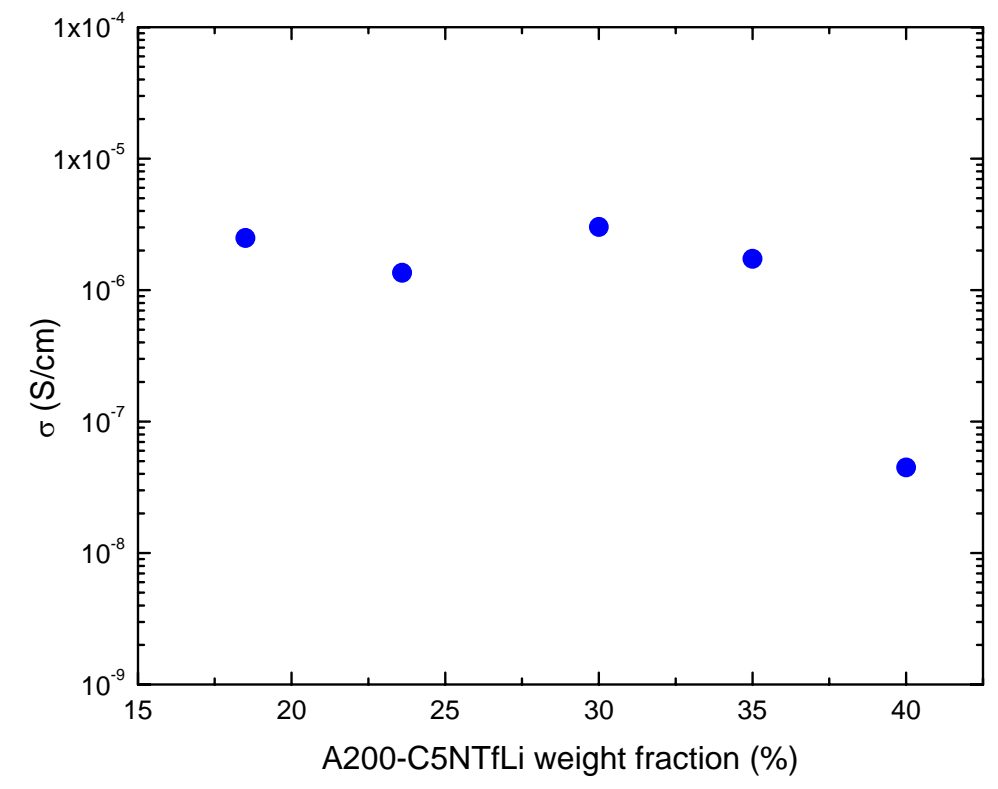

Figure 5. Conductivity of A200-C5NtfLi/polyethylene glycol dimethyl ether (ca. 500 $\mathrm{g} / \mathrm{mol}$ ). Composites as function of A200-C5NtfLi weight fraction. 


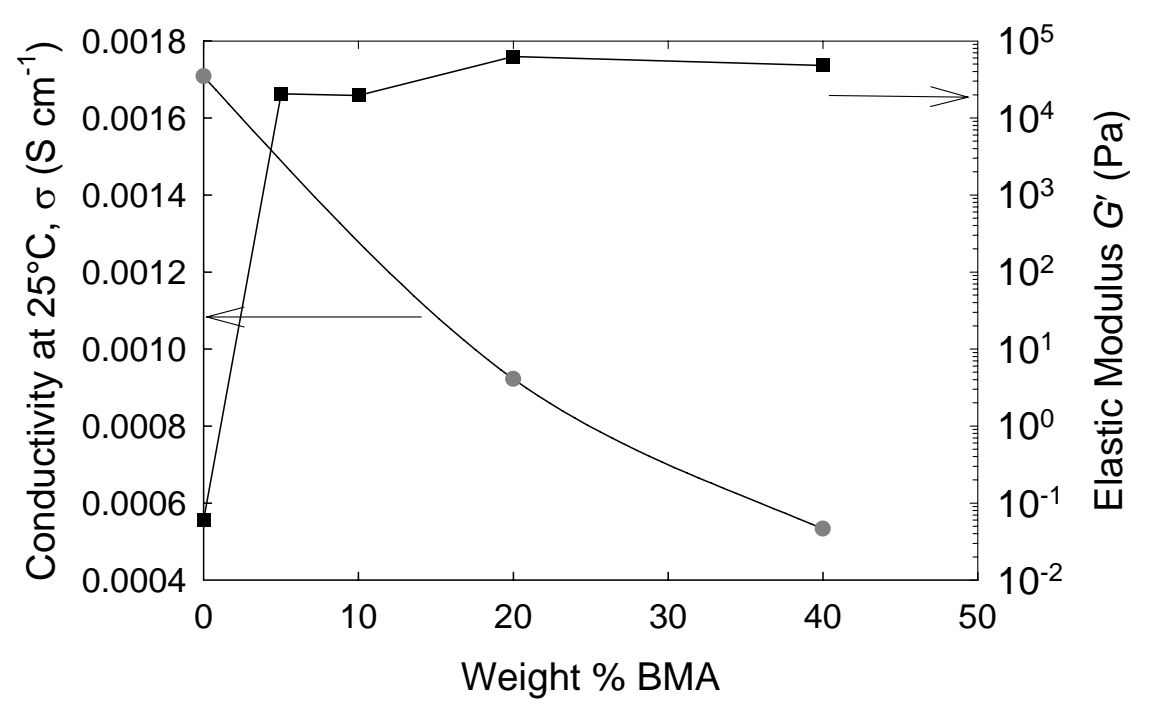

Figure 6. Room-temperature conductivity and elastic moduli $\left(\omega=1 \mathrm{rad} \mathrm{s}^{-1}\right)$ of crosslinked CPEs as a function of butyl methacrylate (BMA) weight percent.

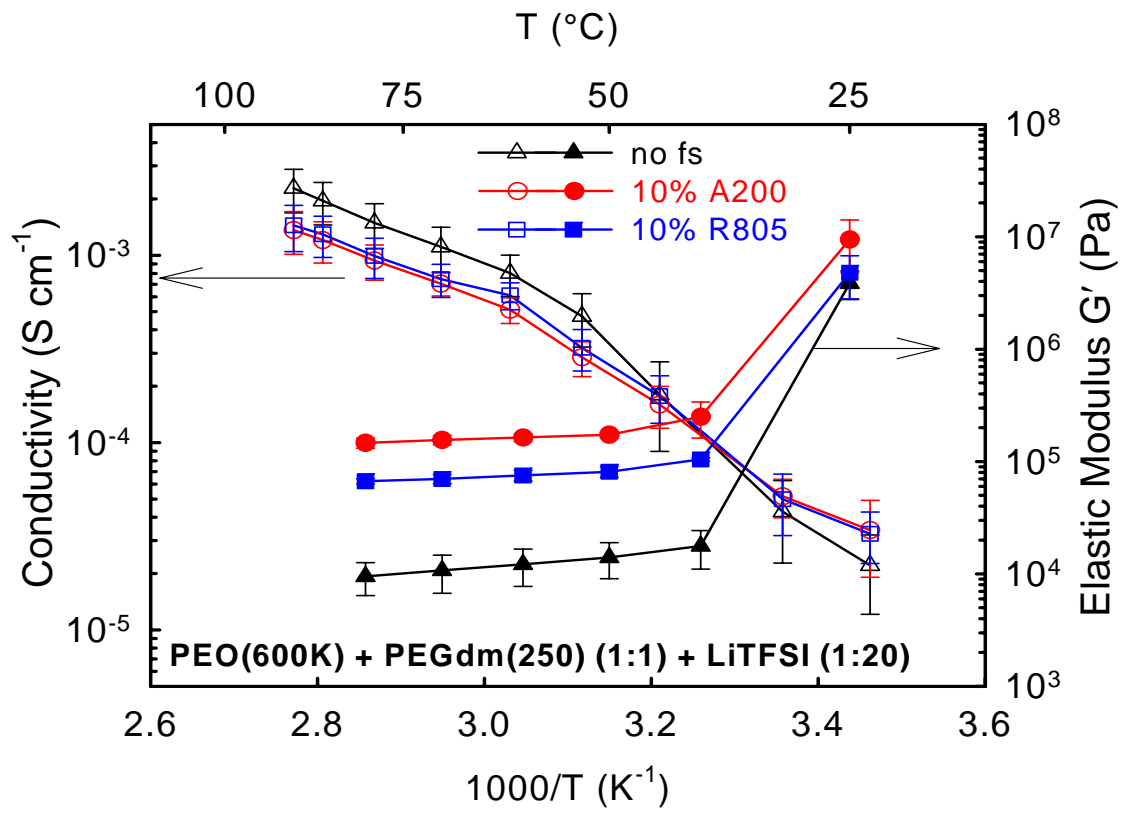

Figure 7. Effect of temperature on the elastic modulus and conductivity of mixed molecular weight systems. 

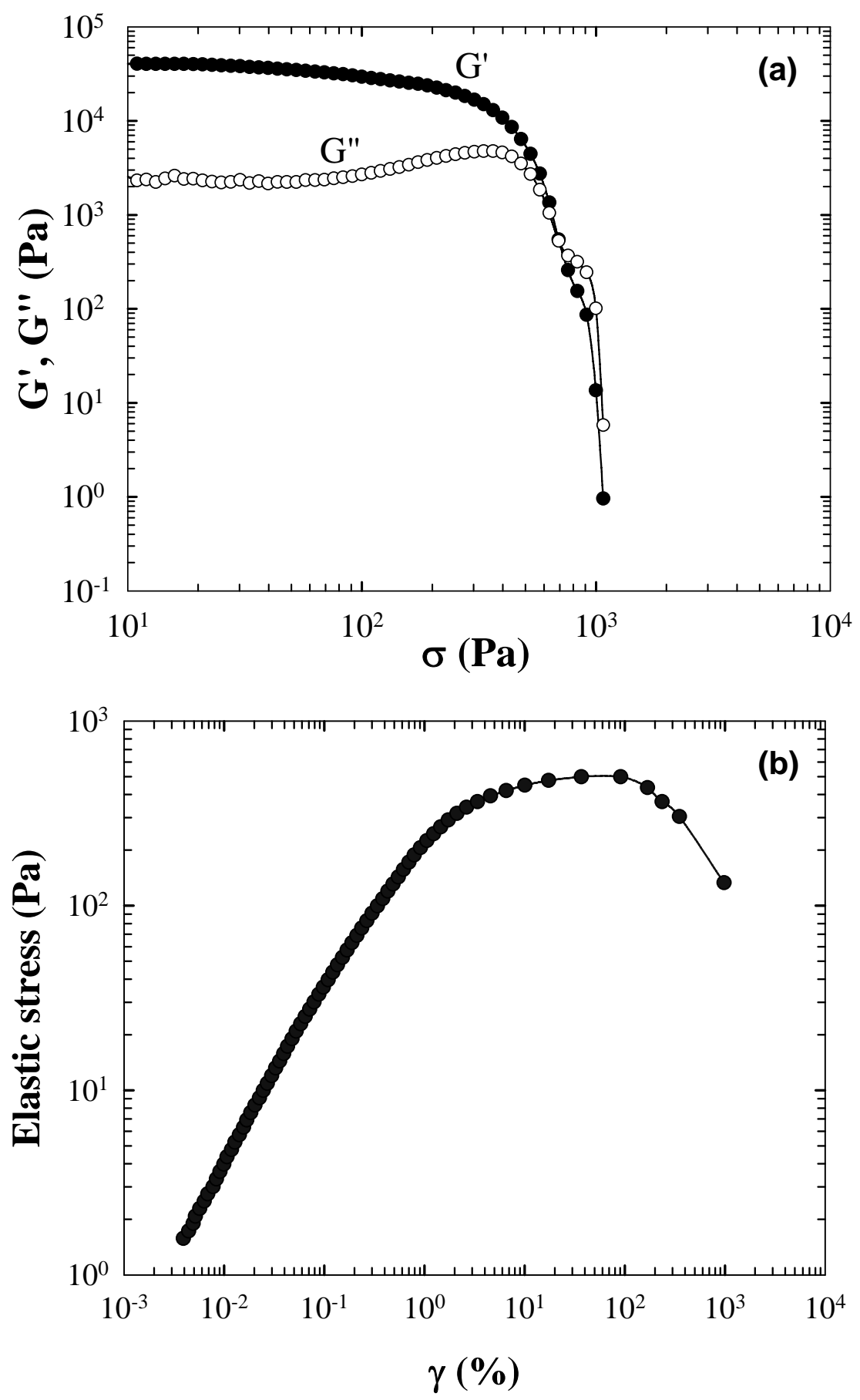

Figure 8. Dynamic stress data for the sample containing 1.07 M LiTFSI in PEGdm 250 and fumed silica volume fraction $(\phi) \sim 0.051$. (a) Dynamic stress sweep showing fumed silica network structure rupturing at high stresses. (b) The elastic stress $\left(G^{\prime} \gamma\right)$ plotted as a function of strain amplitude. The maximum in the elastic stress curve is interpreted as the yield stress of the material. 

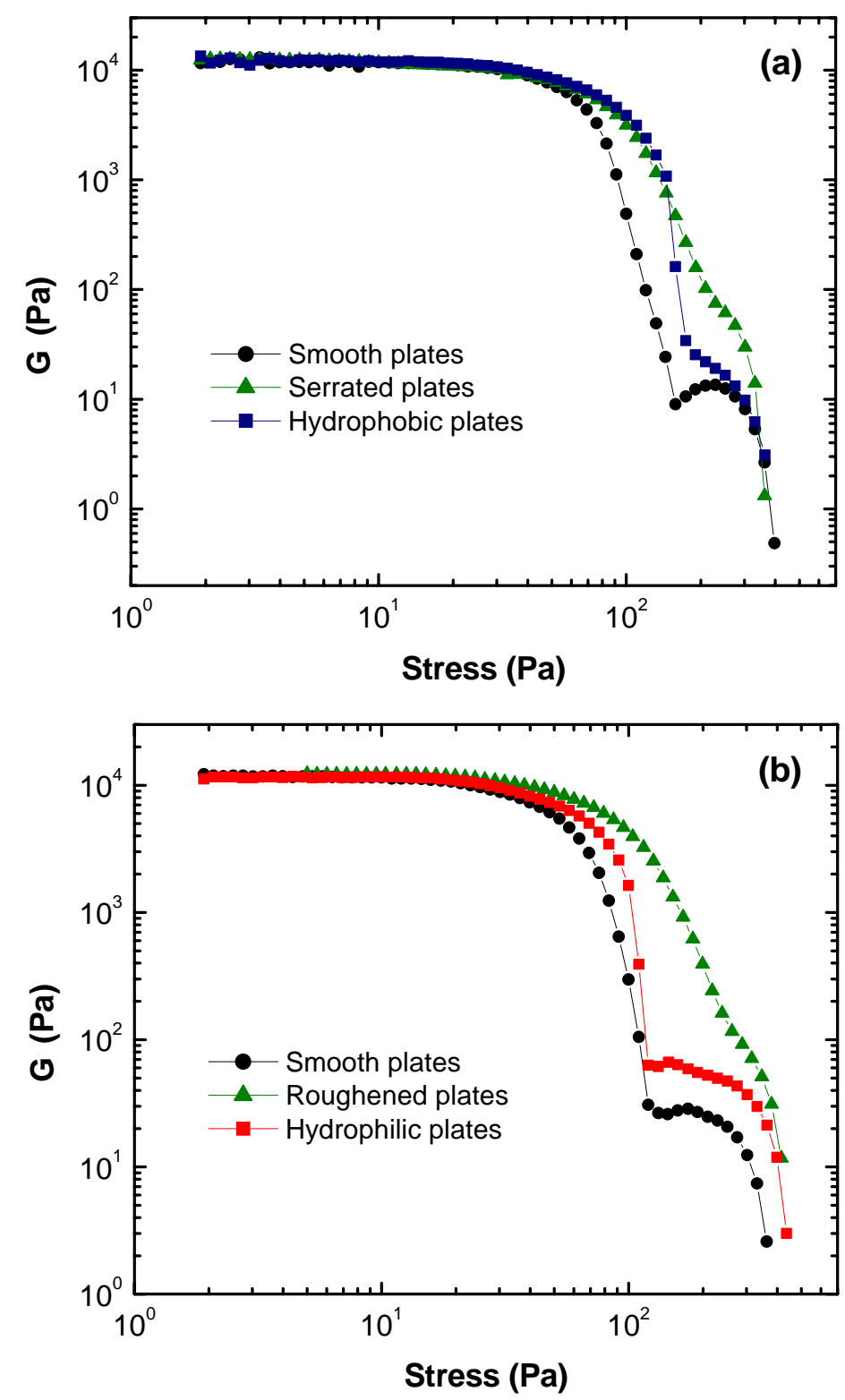

Figure 9. Dynamic stress sweeps for PEGdm(250) + 10wt\% hydrophobic fumed silica (R805) in (a) hydrophobic surface plates, and (b) hydrophilic surface plates. 


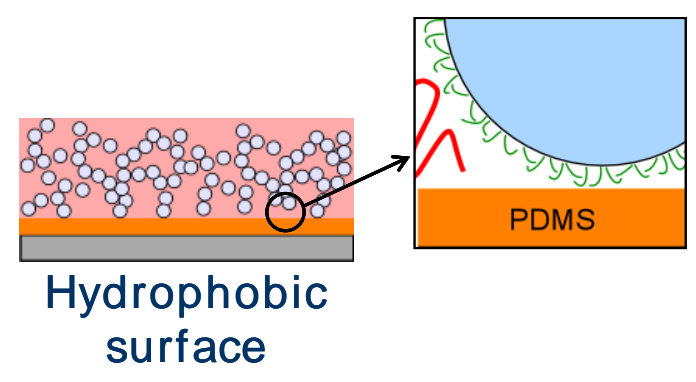

(a)

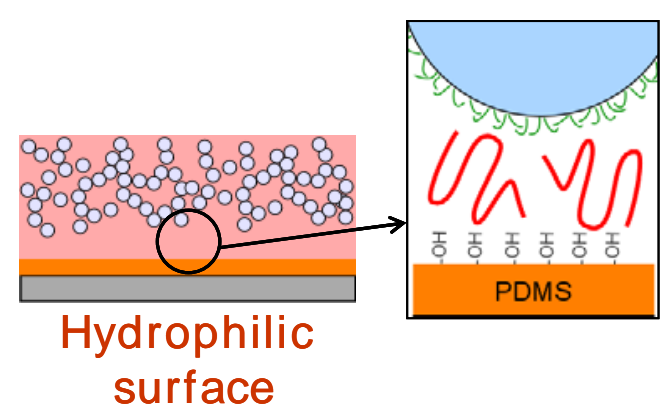

(b)

Figure 10. Schematic of the proposed mechanism for wall slip prevention in surfacemodified plates, a) hydrophobic, and b) hydrophilic plates. The material contains hydrophobic fumed silica. 